\title{
20(s)-ginsenoside Rh2 promotes TRAIL-induced apoptosis by upregulating DR5 in human hepatocellular carcinoma cells
}

\section{Wenmo Liu}

Jilin University

\section{Siqi Wang}

Jilin University

Qinchuan Yang

Jilin University

\section{Xinyao Feng}

Jilin University

Bin Yu ( $\nabla$ yubin@jlu.edu.cn )

Jilin University https://orcid.org/0000-0002-3533-4049

Xianghui Yu

Jilin University

\section{Research Article}

Keywords: cancer therapy, TRAIL, 20(s)-ginsenoside Rh2, DR5, apoptosis

Posted Date: October 29th, 2021

DOI: https://doi.org/10.21203/rs.3.rs-999155/v1

License: (1) This work is licensed under a Creative Commons Attribution 4.0 International License. Read Full License 


\section{Abstract}

Tumor necrosis factor-related apoptosis-inducing ligand (TRAIL) is a potential therapeutic anti-cancer drug with selective cytotoxicity in cancer cells. However, in multiple clinical trials, the therapeutic effect of TRAIL is limited owing to tumor resistance. The combination of small molecules or other drugs may represent a suitable strategy to overcome TRAIL resistance. This study found that 20(s)-ginsenoside Rh2 sensitized non-sensitive human hepatocellular carcinoma cells to TRAIL-induced apoptosis. The combination of TRAIL and Rh2 decreased cell viability and increased caspase cascade-induced apoptosis in several liver cancer cell lines. Moreover, we found that Rh2 reduced the apoptosis-related protein XIAP and Survivin, a negative regulator of the apoptosis pathway. At the same time, Rh2 can further enhance TRAIL-induced apoptosis by upregulating the death receptor 5 (DR5), thereby significantly enhancing its anti-tumor effect. Furthermore, Rh2 enhanced the therapeutic efficacy of TRAIL in mouse xenograft models, suggesting that Rh2 also sensitizes TRAIL in vivo. Taken together, our study indicates that Rh2 may act as a sensitizer in combination with TRAIL to increase the efficacy of its anti-tumor activity.

\section{Introduction}

Hepatocellular carcinoma (HCC) is the seventh most common cancer presenting with solid tumors and the fourth leading cause of cancer-related deaths worldwide [1]. At present, classical chemotherapy is still the main treatment for HCC. Procedures such as surgical resection, ablation, transarterial chemoembolization, and stereotactic body radiation therapy for HCC are current therapeutic strategies [2]. A multidisciplinary approach is associated with improved survival of hepatocellular carcinoma patients, but selecting an appropriate therapeutic strategy is also important for patients [3].

TRAIL, a tumor necrosis factor-related apoptosis-inducing ligand, is an attractive agent for cancer therapy because it induces apoptosis in cancer cells but not in normal cells [4-6]. TRAIL interacts with TRAILR1/death receptor 4 (DR4) and TRAIL-R2/death receptor 5 (DR5) expressed by tumor cells, thereby inducing the apoptotic pathways in cells [7]. There are two principal signaling pathways involved in apoptosis: the extrinsic and intrinsic pathways. The extrinsic pathway is activated when TRAIL binds to death receptors, such as DR4 and DR5, and triggers the caspase cascade. The binding of TRAIL to DR4 and DR5 results in the death-inducing signaling complex (DISC) assembly, which incorporates the adaptor protein Fas-associated death domain (FADD). Through FADD, activation of pro-caspases 8 and 10 lead to the release of active caspase molecules, which activate the effector caspase-3 [8-10]. The intrinsic pathway includes the interplay between members of the Bcl-2 family, leading to the activation of caspase 9 and the effector caspase from mitochondria to the cytoplasm [11-13]. However, not all malignancies are sensitive and responsive to TRAIL. For TRAIL-insensitive tumor cells, a broader approach is to combine other drugs for treatment $[14,15]$. Importantly, such combinations increase the sensitivity to TRAIL, which makes TRAIL play a critical role in cancer therapy. 
Ginsenoside Rh2, a purified ginseng saponin, exhibits anti-tumor effects in various cancer cell types, such as human breast cancer MCF-7 cells [16], human AML THP-1 cells [17], and SK-Hep-1 human hepatoma cells [18]. However, the molecular mechanism of Rh2 anti-tumor activity has not been explained fully, it is mainly related to tumor cell proliferation or apoptosis [19]. For example, Rh2 effectively inhibited IL-6induced signal transducer and activator of transcription 3 (STAT3) phosphorylation and the expression of matrix metalloproteinases (MMPs), resulting in the inhibition of human colorectal cancer cell invasion [20]. In HepG2 cells, Rh2 could suppress proliferation, promote apoptosis, and inhibit metastasis by downregulating $\beta$-catenin through activating GSK-3 $\beta$ [21]. In addition, Rh2 was used in combination with other drugs for synergistic treatment. Rh2 and sodium selenite combination have a synergistic effect on HCT116 human colorectal carcinoma cells [22]. In a previous study, the ginsenoside metabolite Rg3 was combined with TRAIL in treating hepatocellular carcinoma, thus providing a basis for combining TRAIL and $\mathrm{Rh} 2$ [23].

This study found that the combination of TRAIL and Rh2 produced significant anti-tumor effects both in vitro and in vivo. Some cancer cells, including HCC cells, are resistant to TRAIL. However, Rh2-induced TRAIL was more sensitive to the HepG2 cells. Then, we demonstrated that the combined mechanism of TRAIL and Rh2 is to upregulate tumor cells DR5 expression and decrease expression of the apoptosis inhibitory proteins XIAP and Survivin, thereby causing a stronger apoptotic activity of TRAIL, and also verified this hypothesis in vivo. This study will improve the efficiency of treating TRAIL-insensitive cell lines and avoid high-dose drug toxicity while achieving better therapeutic outcomes.

\section{Materials And Methods \\ 2.1. Materials}

Ginsenoside Rh2 ( $\geq 98 \%$ purity, lot number MB6871) was purchased from Dalian Meilun Biotechnology Co., Ltd. (Dalian, China). Dulbecco's modified Eagle's medium (DMEM), RPMI 1640, fetal bovine serum (FBS), penicillin, streptomycin, and trypsin were purchased from Gibco (Gaithersburg, MD, USA).

Antibodies against caspase-3, cleaved caspase-3, and Survivin were obtained from Cell Signaling Technology (Danvers, MA, USA). Caspase-8, XIAP, and Bcl-2 were purchased from BD Biosciences (San Jose, CA, USA). Antibodies against DR4 and DR5 were purchased from Abcam (Cambridge, UK). PARP was purchased from Sino Biological (Beijing, China). Tubulin and $\beta$-actin were purchased from Absin (Shanghai, China).

\subsection{Cell viability assay}

Human hepatocellular carcinoma cells HepG2, SMMC-7701, LM-3, and human liver cells HL7702 were purchased from the American Type Culture Collection (ATCC, Manassas, VA, USA). Cells were cultured with $10 \%$ FBS in DMEM or RPMI 1640 plus $100 \mathrm{U} / \mathrm{mL}$ penicillin and $100 \mathrm{mg} / \mathrm{mL}$ streptomycin at $37^{\circ} \mathrm{C}$ in a humidified $5 \% \mathrm{CO}_{2}$ atmosphere. Experimental cells $\left(1 \times 10^{4} / 100 \mu \mathrm{L}\right)$ were grown in a 96-well plate, 
incubated for 24h, and then treated with TRAIL and Rh2 at the indicated concentrations. Cell viability was assessed using the MTT assay, according to previously described procedures.

\subsection{Annexin V-FITC/PI flow cytometry assay}

Cells were seeded at a concentration of $2 \times 10^{5}$ cells/well in a 6-well plate and incubated with TRAIL and Rh2 for $24 \mathrm{~h}$. Importantly, Rh2 was pretreated for 30 min followed by TRAIL in the combination group. Apoptotic cells were detected using an Annexin V-FITC/PI Apoptosis Detection Kit (KeyGEN BioTECH, Jiangsu, China). Following digestion, the cells were washed twice in ice-cold phosphate-buffered saline (PBS) by centrifugation at $300 \times \mathrm{g}$ for $5 \mathrm{~min}$ and resuspended in $500 \mu \mathrm{L}$ binding buffer. Cells were then stained with $5 \mu \mathrm{L}$ Annexin V-FITC and $5 \mu \mathrm{L}$ propidium iodide (PI) for 15 min in the dark at room temperature and analyzed by two-color flow cytometry (Becton Dickinson, Franklin Lakes, NJ, USA). Annexin V-FITC fluorescence was detected in FL-1, and PI was detected in FL-3.

\subsection{Western blotting}

The harvested cells were mixed with radio immunoprecipitation assay (RIPA) buffer (Beyotime, Jiangsu, China) for $10 \mathrm{~min}$. The proteins were separated by SDS-PAGE and transferred onto a nitrocellulose membrane (Millipore, Billerica, MA, USA) after electrophoresis. Then, the membranes were incubated for $1 \mathrm{~h}$ at room temperature in blocking buffer, followed by overnight incubation at $4^{\circ} \mathrm{C}$ with primary antibodies overnight. The next day, the membranes were washed with PBS containing $0.2 \%$ Tween 20 (PBST) and incubated with secondary antibodies for $1 \mathrm{~h}$. After washing three times with PBST, the signal was detected using an automatic chemiluminescence image analysis system (Tanon, Shanghai, China).

\subsection{Caspase $-3,-8$, and -9 activity analysis}

Caspase activity was measured using a caspase activity kit (Beyotime, Jiangsu, China) according to the manufacturer's instructions. The detailed method was described previously. Briefly, cells were incubated with TRAIL and Rh2 for 4, 8, 16, and 24h, respectively. Rh2 was pretreated for 30 min, followed by TRAIL in the combination group. The cells were resuspended in lysis buffer and incubated on ice for $15 \mathrm{~min}$. The lysate was centrifuged at $12,000 \mathrm{rpm}$ at $4^{\circ} \mathrm{C}$ for $15 \mathrm{~min}$. Activities of caspase- $3,-8$, and -9 were measured using the substrate peptides Ac-DEVD-pNA, Ac-IETD-pNA, and Ac-LEHD-pNA, respectively. The release of pNA was determined by measuring the absorbance at a wavelength of $405 \mathrm{~nm}$.

\subsection{Human apoptosis protein array}

We used an apoptosis array purchased from R\&D Systems (Minneapolis, MN, USA) to detect different apoptotic proteins. Briefly, HepG2 cells were treated with $25 \mu \mathrm{M}$ Rh2 for $24 \mathrm{~h}$. Approximately $200 \mu \mathrm{g}$ of protein lysate were used according to the manufacturer's instructions. Apoptotic proteins were visualized using enhanced chemiluminescence (ECL).

\subsection{Reverse transcription PCR}

HepG2 cells were treated with TRAIL ( $50 \mathrm{ng} / \mathrm{mL})$, Rh2 (25 $\mu \mathrm{M})$, and Rh2 pretreated for 30 min, followed by TRAIL in the combination group. The TransZol Up Plus RNA Kit (Transgen, Beijing, China) was used to 
extract total RNA from treated cells according to the manufacturer's instructions. cDNA was obtained using the PrimeScript RT Reagent Kit with gDNA Eraser (Takara, Otsu, Japan). Using cDNA as the template, a quantitative real-time PCR assay was performed with SYBR Premix ExTaq (Takara) and a CFX96 real-time PCR system (Bio-Rad, CA, USA). $\beta$-actin was used as the reference gene.

\subsection{Flow cytometry analysis}

For the HepG2 cells stained for DR4 and DR5, the cells were suspended in PBS after Rh2 treatment for 12 h. Cells were then incubated with PE anti-human CD261 (DR4, TRAIL-R1) antibody (BioLegend, CA, USA) and APC anti-human CD262 (DR5, TRAIL-R2) antibody (BioLegend, CA, USA) for 20 min in the dark. Subsequently, the cells were washed with PBS and analyzed using flow cytometry (Becton Dickinson, Franklin Lakes, NJ, USA).

Tumor tissues were collected in RPMI 1640 and cut into several pieces. The cells were then ground into individual cells in a plate after $1 \mathrm{~h}$ of collagenase treatment at $37^{\circ} \mathrm{C}$. The cells were centrifuged at $300 \times \mathrm{g}$ for 5 minutes, washed with PBS, and resuspended in staining buffer. Mouse intratumoral cells were stained with the following antibodies: FITC anti-human CD45, PE anti-human CD261 (DR4, TRAIL-R1), APC anti-human CD262 (DR5, TRAIL-R2), and isotype control FITC Mouse IgG1 (BioLegend, CA, USA) for 20 min in the dark. The stained cells were analyzed using flow cytometry.

\subsection{Xenograft mouse models}

Female BALB/c nu/nu mice (5 weeks old) were obtained from Vital River Laboratories (VRL, Beijing, China). Human hepatocellular carcinoma cells HepG2 $\left(5 \times 10^{5}\right)$ in the log phase were implanted subcutaneously (s.c.) in the flanks of mice. The mice were randomized into four groups (PBS, TRAIL, Rh2, and TRAIL $+\mathrm{Rh} 2, \mathrm{n}=10$ /group). When the tumor reached a volume of approximately 100-150 $\mathrm{mm}^{3}$, mice were intraperitoneally administered a dose of $1 \mathrm{mg} / \mathrm{kg} \mathrm{Rh} 2,10 \mathrm{nM}$ TRAIL, or PBS $(100 \mu \mathrm{L})$ for eight consecutive days. Tumor growth was monitored daily for 20 days, and the tumor volume $\left(\mathrm{mm}^{3}\right)$ was calculated as (length $\times$ width $\left.^{2}\right) / 2$. When the total tumor volume reached $1,500 \mathrm{~mm}^{3}$, the animals were euthanized. At the end of treatment, three mice from each group were sacrificed randomly. The liver, kidney, and tumor tissues were fixed with $4 \%$ paraformaldehyde, and paraffin sections were obtained for H\&E staining and immunohistochemistry.

\subsection{Statistical analysis}

Values are expressed as the mean \pm SEM. Statistical analysis was performed using a one-way analysis of variance or an unpaired $t$-test followed by the Newman-Keuls test. Statistical significance was set at $P$ $<0.05$. Statistical analysis was performed using GraphPad Prism 8.

\section{Results}

\subsection{Rh2 sensitizes human hepatocellular carcinoma cells to TRAIL-induced cell death}


TRAIL may have produced anti-tumor effects in various tumor cells in earlier studies by inducing apoptosis. The cytotoxic activity of TRAIL and Rh2 in human hepatocellular carcinoma cells was assessed using the MTT assay. The results showed that a concentration of $200 \mathrm{ng} / \mathrm{mL}$ of TRAlL alone was required for cytotoxicity. When the TRAIL concentration was $200 \mathrm{ng} / \mathrm{mL}$, the cell viability of SMMC7701 was $42.8 \%$, but that of HepG2 and LM-3 cells was approximately $80 \%$ (Fig. 1a). Rh2 concentrations greater than $25 \mu \mathrm{M}$ were required for substantial cytotoxicity in all three cell lines (Fig. 1b). However, when cells were pretreated with Rh2 for 30 min followed by low concentrations of TRAlL $(25 \mathrm{ng} / \mathrm{mL})$ for $16 \mathrm{~h}$, the SMMC-7701, and LM-3 cells were underwent cell death as evidenced by MTT assay (Fig. 1c). With the same treatment, the cell viability in the combination of TRAIL and $50 \mu \mathrm{M} \mathrm{Rh2}$ was $11 \%$, which was remarkably suppressed compared with cells treated with Rh2 and TRAIL alone (Fig. 1d). After the combination treatment, the inhibitory effect of TRAIL on HepG2 cells was relieved. Moreover, the combined group cells showed changes in cell morphology, and cell shrinkage and death occurred (Fig. 1e). However, human normal liver cells (HL7702) were insensitive to TRAIL and Rh2, indicating that sensitization may be limited to cancer cells (Fig. 1f).

\subsection{Rh2 promotes TRAIL-induced caspase-dependent apoptotic cell death}

To evaluate the effect of TRAIL and Rh2 on apoptosis in HepG2 cells, we performed an experiment to detect the apoptotic pathway. The results showed that the number of apoptotic cells in the TRAIL and Rh2 combined treatment was significantly higher than those in the Rh2 and TRAIL alone groups (Fig. 2a and 2 b). TRAIL-induced apoptosis is executed by the extrinsic cell death pathway, with caspase- 3 as the executioner caspase. Rh2 alone did not affect caspase-3 cleavage in HepG2 cells, but Rh2 pretreatment significantly augmented TRAIL-induced cleavage of caspase-3 and PARP cleavage. Meanwhile, caspase8 was also activated in the combined TRAIL and Rh2 treatment group (Fig. 2c and 2d). To further illustrate the effect of the caspase pathway, we detected caspase-3, -8 , and -9 enzyme activity at different time points. The results showed that caspase- 3 and -8 enzyme activity peaked at $4 \mathrm{~h}$ after combined TRAIL and Rh2 treatment, and caspase activity was significantly stronger than Rh2 alone (Fig. 2e). However, there was no apparent effect on caspase- 9 activity, whether in the combined or the separate treatment groups (Fig. 2e), indicating that the synergistic effect of TRAIL and Rh2 on apoptosis may be mediated through a caspase-dependent pathway.

\subsection{Rh2 downregulates inhibitor of apoptosis proteins}

Based on the above results, Rh2 alone can also induce apoptosis in liver cancer cells. To confirm the mechanism of Rh2 apoptosis, we used a human apoptosis protein array as shown in Fig. 3a and 3b, which demonstrated the upregulation of Bad, cleaved caspase-3, DR5, Fas, and SMAC/Diablo. Moreover, the detection of apoptosis proteins revealed that Survivin expression was suppressed. Next, we detected the expression of apoptosis inhibition-associated proteins using immunoblotting to confirm the effect of $\mathrm{Rh} 2$ on the apoptotic pathway. As shown in Fig. 3c, Rh2 decreased XIAP and Bcl-2 protein levels, indicating that $\mathrm{Rh} 2$ promotes apoptosis by downregulating apoptosis-inhibiting proteins such as XIAP. In addition, when TRAIL and Rh2 were combined, the protein expression of XIAP and Survivin decreased as 
the Rh2 concentration increased (Fig. 3d). Taken together, our results demonstrate that Rh2 can induce apoptosis of liver cancer cells by downregulating the expression of XIAP and Survivin, and TRAIL does not affect this process.

\subsection{Rh2 sensitizes TRAIL-induced apoptosis via DR5 upregulation}

DR4 and DR5 expression directly affect the TRAIL-induced apoptosis pathway. Quantitative real-time PCR was used to measure DR4 and DR5 mRNA expression (Fig. 4a). After Rh2 treatment, increased DR5 mRNA levels were observed, and DR4 mRNA levels remained unchanged. Consistent with the mRNA changes, Rh2 markedly induced the expression of DR5 from $2 \mathrm{~h}$ onwards, whereas DR4 expression did not change significantly (Fig. 4b). Furthermore, we examined the expression of DR4 and DR5 after Rh2 treatment in HepG2 cells using flow cytometry analysis, as shown in Fig. 4c. The flow cytometry results also showed that DR5 was significantly upregulated compared with the control, but DR4 expression was unchanged. The relative mean optical density of DR5 in the Rh2-treated group increased significantly (Fig. 4d), indicating that Rh2 may promote TRAlL-induced apoptosis of liver cancer cells by upregulating DR5.

\subsection{Combination treatment with Rh2 and TRAIL potentiates in vivo anti-hepatocellular carcinoma activity}

The anti-tumor effects of Rh2 and TRAIL against HCC were further investigated in xenograft mice. HepG2 cells were inoculated into the flanks of nude mice, and when tumors were measurable, mice were matched for tumor volumes and assigned to TRAIL, Rh2, or a combination of TRAIL and Rh2. Tumor volumes in the TRAIL, Rh2, and Rh2-TRAIL combination groups were approximately $98 \%, 86 \%$, and $36 \%$, respectively, compared with the control group (Fig. 5a). It is worth noting that the combination of Rh2 and TRAIL inhibited tumor growth compared with the control group and inhibited tumor growth compared to Rh2 or TRAIL alone. To further explore whether the tumor-suppressive effect in vivo is due to DR5 upregulation in tumor cells by Rh2, we evaluated the intratumoral expression of DR5. The upregulation of DR5 in mice treated with Rh2 or the combination of TRAIL and Rh2 (Fig. 5b) suggests that Rh2 upregulates DR5, which causes TRAIL to induce tumor cell apoptosis and inhibit tumor growth. Next, we investigated the effect of the treatments on in vivo apoptosis by examining cleaved caspase-3 immunohistochemistry staining and H\&E staining of xenografted tumors. Caspase- 3 cleavage was more pronounced in tumor sections from mice treated with TRAIL plus Rh2 than in tumors from mice treated with either TRAlL or Rh2 alone (Fig. 5c). Quantitation of cleaved caspase-3 staining in tumor sections indicated a significant increase in cell death in the tumors of the mice on combination treatment compared with either treatment alone (Fig. 5d). Importantly, no significant changes were observed during therapy in the livers and kidneys of mice of any treatment group (Fig. 5e), indicating that TRAIL or Rh2 is generally well-tolerated in vivo.

\section{Discussion}


As a novel and promising anti-cancer drug, TRAIL is becoming a very useful strategy for treating cancer [24]. However, TRAIL-based therapy still needs to overcome some obstacles due to acquired or intrinsic drug resistance. In this study, we suggest that Rh2 could act as a sensitizer to TRAIL in HepG2 cells. We found that Rh2-mediated DR5, Bcl-2, XIAP, and Survivin regulation in TRAIL sensitization. Rh2 increased DR5 expression at the mRNA level and downregulated XIAP and Survivin expression. Furthermore, we verified that Rh2 upregulates the expression of DR5 and that combination therapy has a stronger antitumor effect than Rh2 alone in mouse xenograft models.

We show that Rh2 can enhance TRAIL-induced tumor apoptosis in insensitive HepG2 cells by upregulating DR5 expression. Although most cancer cells express DR4 and DR5, the expression level of receptors plays a critical role in determining cell fate in response to TRAIL. Numerous studies have shown convincing evidence that DR5 upregulation can enhance the sensitivity of TRAIL-induced apoptosis [25, 26]. Regulate the apoptotic activity of TRAIL by controlling the expression of DR5. Previous studies have demonstrated that telmisartan can sensitize TRAIL and enhance NSCLC tumor cell death [27]. The molecular mechanism includes the generation of ROS, leading to DR5 upregulation. Wu et al. reported enhanced sensitivity to TRAIL therapy following luteolin treatment in NSCLC cells, which may be associated with increased DR5 expression and upregulation of Drp1-mediated mitochondrial fission [28]. We found that Rh2 directly increased DR5 mRNA and protein expression and caused subsequent activation of the caspase cascade following TRAIL treatment. The combination of TRAIL and Rh2 mainly activated the caspase-3 and caspase-8 pathways. Yesi et al. showed that NanoTRAIL generated ROStriggered JNK activation and induced subsequent autophagy-assisted DR5 upregulation, resulting in a significantly enhanced anti-tumor efficacy of TRAIL [29]. Therefore, regardless of which compound is combined with TRAIL, it is likely that DR5 plays a major role in the downstream signaling pathway. Meanwhile, we further evaluated the expression of DR5 in the tumor microenvironment in mouse xenograft models. The expression of DR5 in tumor cells was upregulated due to the effect of Rh2, indicating that the regulatory effect of Rh2 in vivo is consistent with the in vitro results. We definitively demonstrated that Rh2 acts as an activator to upregulate DR5 to sensitize tumor cells to TRAIL. However, the molecular mechanism of Rh2 leading to the upregulation of DR5 expression requires further study.

Although upregulating DR5 expression is the key to activating TRAIL activity, regulating downstream signals can maximize TRAIL function. The anti-tumor mechanism underlying the effects of Rh2 is not fully understood, but there are similar mechanisms in different tumor cells. Previously, Wang et al. reported that GRh2 significantly decreased the anti-apoptotic protein Bcl-2 in leukemia cells [30]. We also confirmed that Rh2 treatment reduced the protein expression of Bcl-2 in HepG2 cells, increased the expression of pro-apoptotic proteins Bad and Bax, and reduced levels of XIAP and Survivin. Raedeh et al. showed that XIAP might also play a role in inducing resistance to TRAIL-induced cell death in leukemia [31]. Indeed, XIAP, as a key central component, functions in the crosstalk between the intrinsic and extrinsic pathways. The combination of XIAP inhibitors and TRAIL receptor agonists sensitized TRAILresistant cells to hematological neoplasms. Consequently, inhibiting XIAP expression may make tumor cells more sensitive to TRAIL through the combination of Rh2. 
Collectively, we showed that Rh2 sensitized HepG2 cells to TRAIL-induced apoptosis through DR5 upregulation both in vivo and in vitro and simultaneously regulated apoptosis-related proteins. Our study indicates that the combination of Rh2 and TRAIL can be used as a promising treatment strategy for liver cancer.

\section{Declarations}

\section{Competing interests}

The authors declare that they have no competing interests.

\section{Acknowledgments}

This work was supported by the National Natural Science Foundation of China [grant number 32071466]; the Project in the Science \& Technology Development Plan of Jilin Province [grant number 20190304034YY]; the State Key Laboratory of Cell Biology [grant number SKLCB2018KF007]; and Fundamental Research Funds for Central Universities.

\section{References}

1. Yang, J.D. and J.K. Heimbach, New advances in the diagnosis and management of hepatocellular carcinoma. Bmj, 2020: p. m3544.

2. Couri, T. and A. Pillai, Goals and targets for personalized therapy for HCC. Hepatology International, 2019. 13(2): p. 125-137.

3. Debes, J., et al., Serum Biomarkers for the Prediction of Hepatocellular Carcinoma. Cancers, 2021. 13(7): p. 1681.

4. Wiley SR, S.K., Smolak PJ, Din WS, Huang CP, Nicholl JK, et al., </dentification and characterization of a new member of the TNF family that induces apoptosis .pdf>. Immunity, 1995. 3: p. 673-82.

5. Walczak H, M.R., Ariail K, Gliniak B, Griffith TS, Kubin M, et al., Tumoricidal activity of tumor necrosis factor-related apoptosis-inducing ligand in vivo. Nat Med, 1995. 5: p. 157-63.

6. Ashkenazi A, P.R., Fong S, Leung S, Lawrence DA, Marsters SA, and e. al., Safety and antitumor activity of recombinant soluble Apo2 ligand. J Clin Invest 1999. 104: p. 155-62.

7. Wong, S.H.M., et al., The TRAIL to cancer therapy: Hindrances and potential solutions. Critical Reviews in Oncology/Hematology, 2019. 143: p. 81-94.

8. de Miguel, D., et al., Onto better TRAILs for cancer treatment. Cell Death \& Differentiation, 2016. 23(5):

p. 733-747. 
9. Stuckey, D.W. and K. Shah, TRAIL on trial: preclinical advances in cancer therapy. Trends in Molecular Medicine, 2013. 19(11): p. 685-694.

10. O'Leary, L., et al., Decoy receptors block TRAIL sensitivity at a supracellular level: the role of stromal cells in controlling tumour TRAIL sensitivity. Oncogene, 2015. 35(10): p. 1261-1270.

11. Zhang, L. and B. Fang, Mechanisms of resistance to TRAIL-induced apoptosis in cancer. Cancer Gene Therapy, 2004. 12(3): p. 228-237.

12. Fulda, S. and K.M. Debatin, Extrinsic versus intrinsic apoptosis pathways in anticancer chemotherapy. Oncogene, 2006. 25(34): p. 4798-4811.

13. Czabotar, P.E., et al., Control of apoptosis by the BCL-2 protein family: implications for physiology and therapy. Nature Reviews Molecular Cell Biology, 2013. 15(1): p. 49-63.

14. Han, L., et al., Combination of the natural compound Periplocin and TRAIL induce esophageal squamous cell carcinoma apoptosis in vitro and in vivo: Implication in anticancer therapy. Journal of Experimental \& Clinical Cancer Research, 2019. 38(1).

15. Jo, E.B., et al., Combination therapy with c-met inhibitor and TRAIL enhances apoptosis in dedifferentiated liposarcoma patient-derived cells. BMC Cancer, 2019. 19(1).

16. Lee, H., et al., Ginsenoside Rh2 epigenetically regulates cell-mediated immune pathway to inhibit proliferation of MCF-7 breast cancer cells. Journal of Ginseng Research, 2018. 42(4): p. 455-462.

17. Wang, Z., et al., Enhancing the antitumor activity of an engineered TRAIL-coated oncolytic adenovirus for treating acute myeloid leukemia. Signal Transduction and Targeted Therapy, 2020. 5(1).

18. Oh, J., et al., Caspase-3-dependent protein kinase $C$ delta activity is required for the progression of Ginsenoside-Rh2-induced apoptosis in SK-HEP-1 cells. Cancer Letters, 2005. 230(2): p. 228-238.

19. Nag, S.A., Ginsenosides as anticancer agents: In vitro and in vivo activities, structure-activity relationships, and molecular mechanisms of action. Frontiers in Pharmacology, 2012. 3.

20. S, H., et al., Ginsenoside 20(S)-Rh2 exerts anti-cancer activity through targeting IL-6-induced JAK2/STAT3 pathway in human colorectal cancer cells. Journal of ethnopharmacology, 2016. 194: p. 8390.

21. Shi, Q., et al., Anticancer effect of 20(S)-ginsenoside Rh2 on HepG2 liver carcinoma cells: Activating GSK-3 $\beta$ and degrading $\beta$-catenin. Oncology Reports, 2016. 36(4): p. 2059-2070.

22. Zhu C, L.F., Qian W, Zhang T, Li F., Combined Effect of Sodium Selenite and Ginsenoside Rh2 on HCT116 Human Colorectal Carcinoma Cells. Arch Iran Med, 2016. 19(1): p. 23-29. 
23. Lee, J.-Y., et al., Sensitization of TRAIL-Induced Cell Death by 20(S)-Ginsenoside Rg3 via CHOPMediated DR5 Upregulation in Human Hepatocellular Carcinoma Cells. Molecular Cancer Therapeutics, 2013. 12(3): p. 274-285.

24. Kelley, S., Targeting death receptors in cancer with Apo2L/TRAIL. Current Opinion in Pharmacology, 2004. 4(4): p. 333-339.

25. Jeon, M.-Y., et al., Dexamethasone Inhibits TRAIL-Induced Apoptosis through c-FLIP(L) Upregulation and DR5 Downregulation by GSK3 $\beta$ Activation in Cancer Cells. Cancers, 2020. 12(10): p. 2901.

26. Woo, S.M., K.-j. Min, and T.K. Kwon, Magnolol Enhances the Therapeutic Effects of TRAIL through DR5 Upregulation and Downregulation of c-FLIP and Mcl-1 Proteins in Cancer Cells. Molecules, 2020. 25(19): p. 4591.

27. Rasheduzzaman, M., et al., Telmisartan generates ROS-dependent upregulation of death receptor 5 to sensitize TRAIL in lung cancer via inhibition of autophagy flux. The International Journal of Biochemistry \& Cell Biology, 2018. 102: p. 20-30.

28. Wu, B., et al., Luteolin enhances TRAIL sensitivity in non-small cell lung cancer cells through increasing DR5 expression and Drp1-mediated mitochondrial fission. Archives of Biochemistry and Biophysics, 2020. 692: p. 108539.

29. Shi, Y., et al., Oxidative stress-driven DR5 upregulation restores TRAIL/Apo2L sensitivity induced by iron oxide nanoparticles in colorectal cancer. Biomaterials, 2020. 233: p. 119753.

30. Wang, X. and Y. Wang, Ginsenoside Rh2 Mitigates Pediatric Leukemia Through Suppression of Bcl-2 in Leukemia Cells. Cellular Physiology and Biochemistry, 2015. 37(2): p. 641-650.

31. Saraei, R., et al., The role of XIAP in resistance to TNF-related apoptosis-inducing ligand (TRAIL) in Leukemia. Biomedicine \& Pharmacotherapy, 2018. 107: p. 1010-1019.

\section{Schemes}

Schematic is only available as a download in Supplemental Files section

\section{Figures}




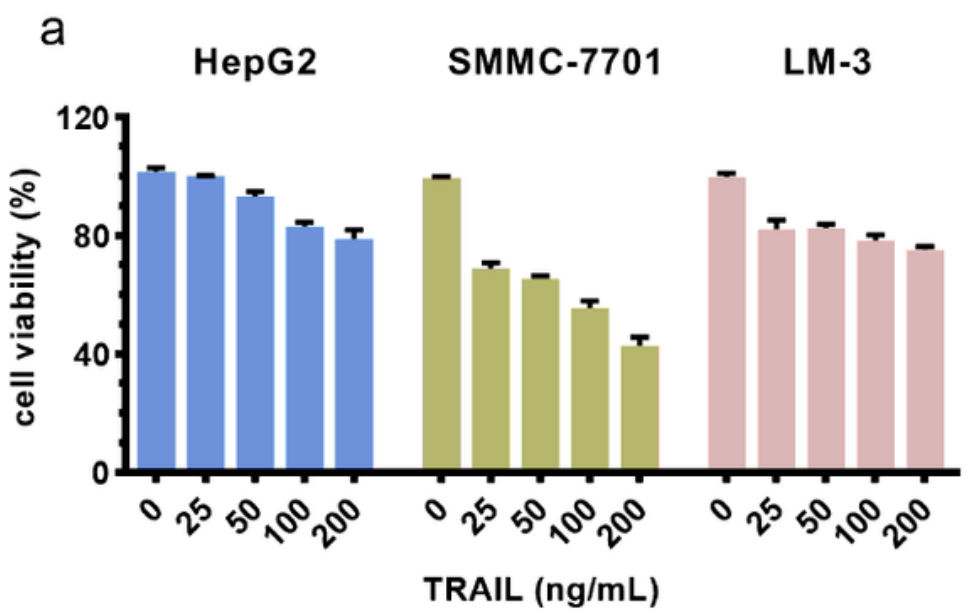

C
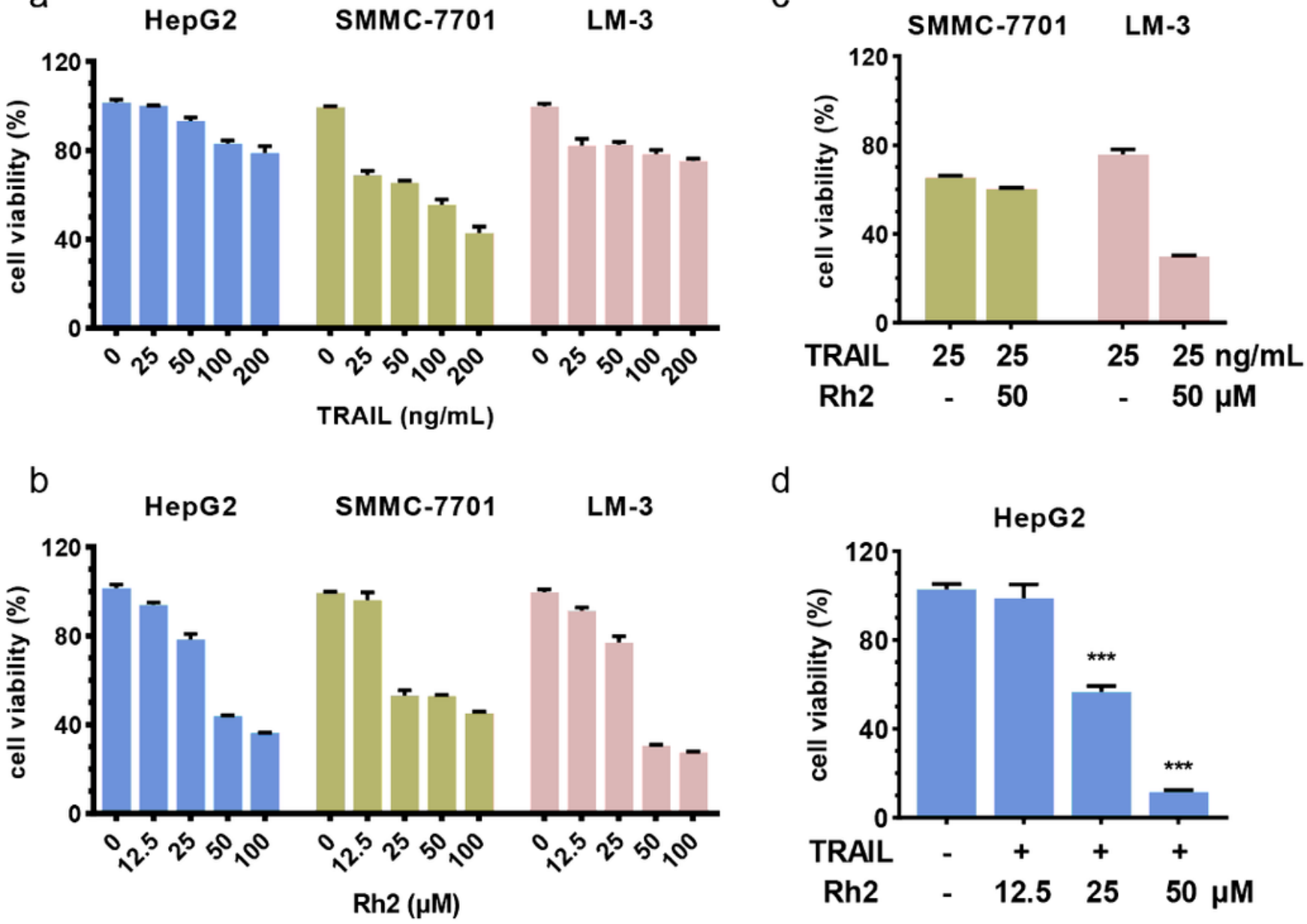

d

e

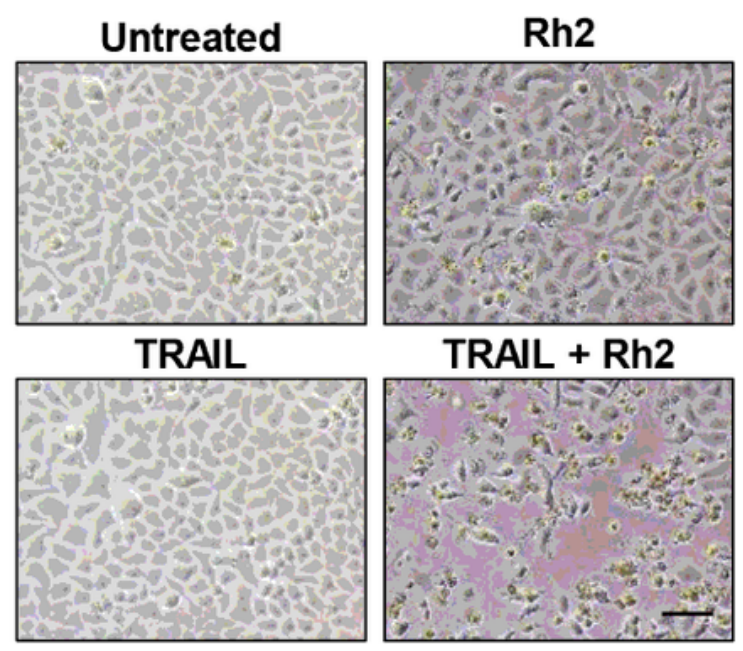

$f$
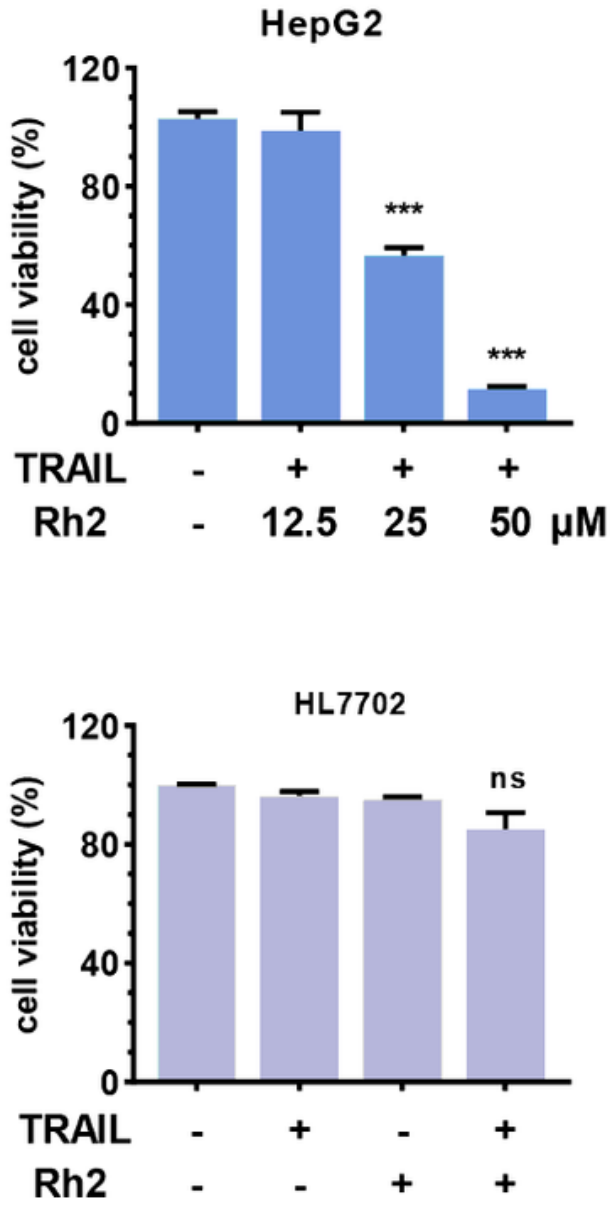

Figure 1

Combined TRAIL and Rh2 treatment in HepG2. 
a b
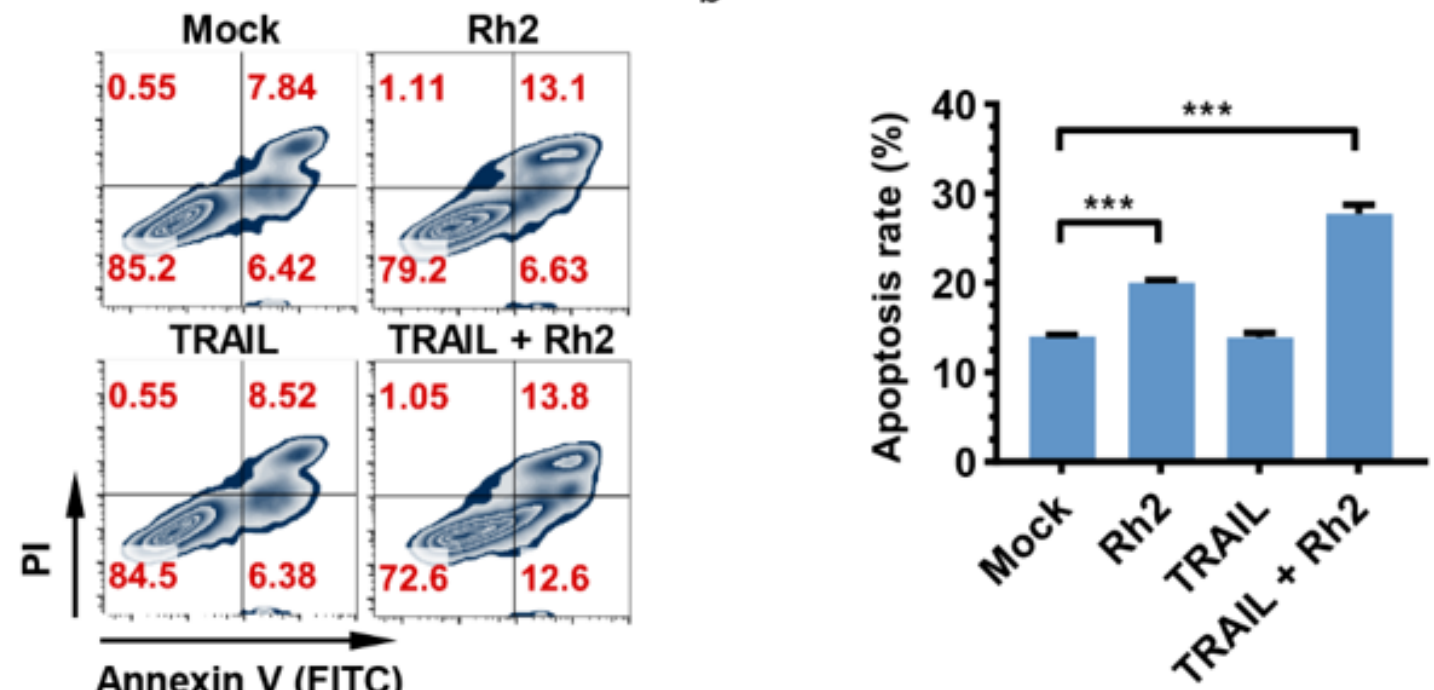

C

Annexin V (FITC)

d

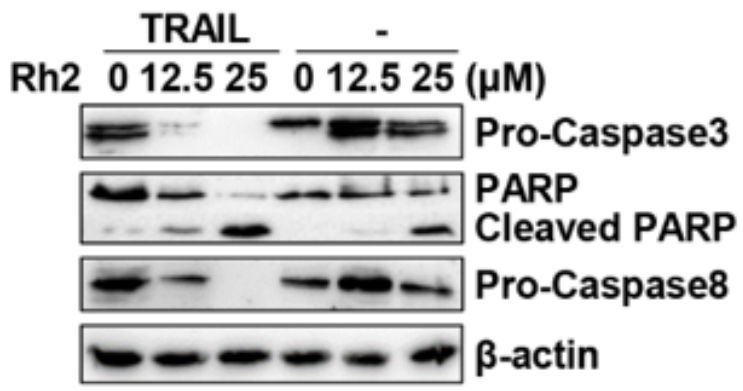

Caspase-3 Caspase-8

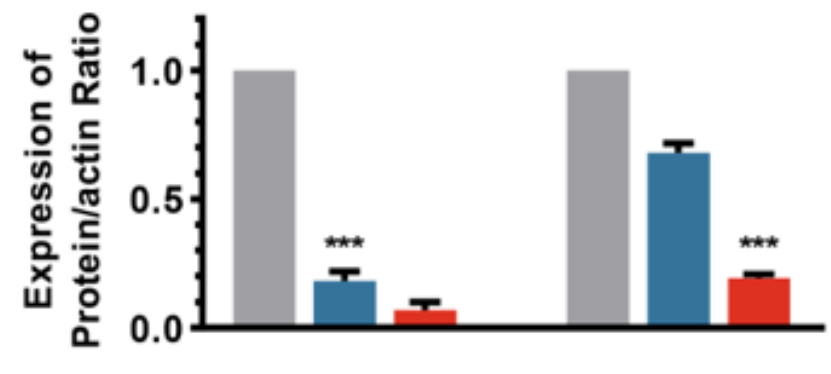

e
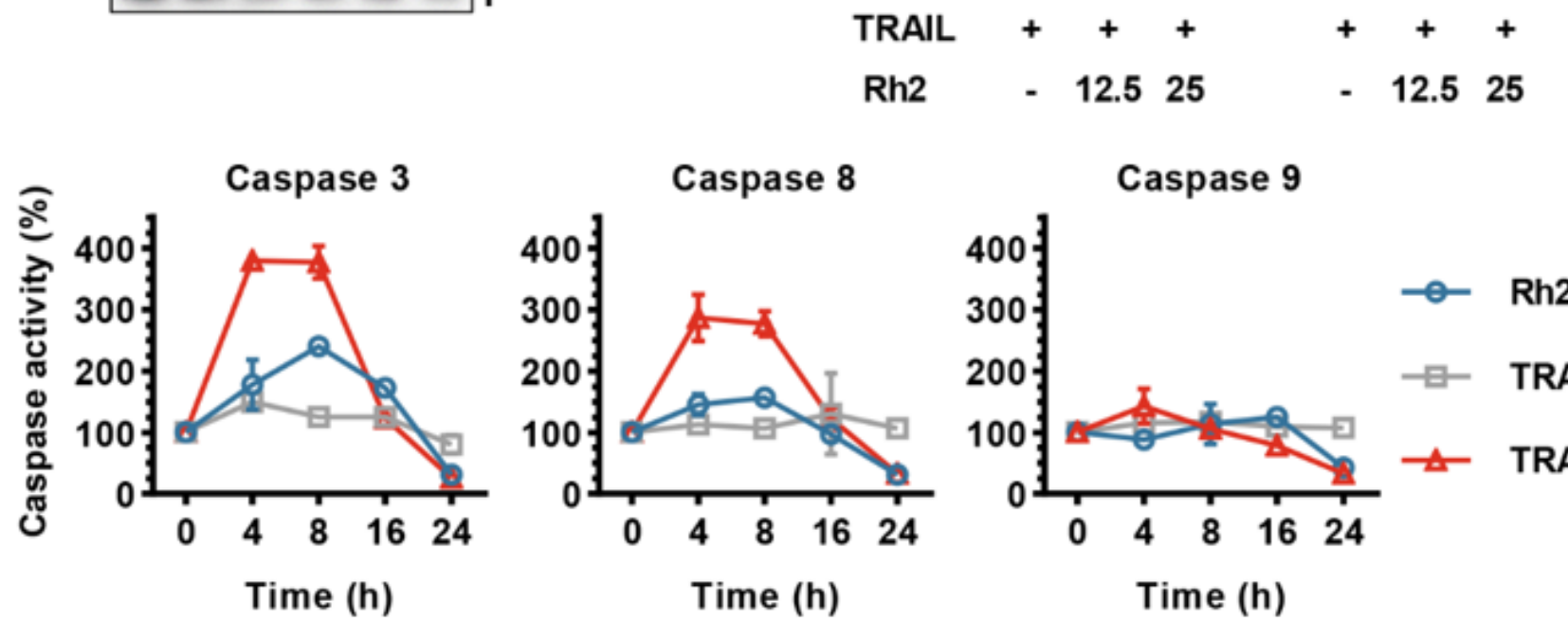

Caspase 8

Caspase 9
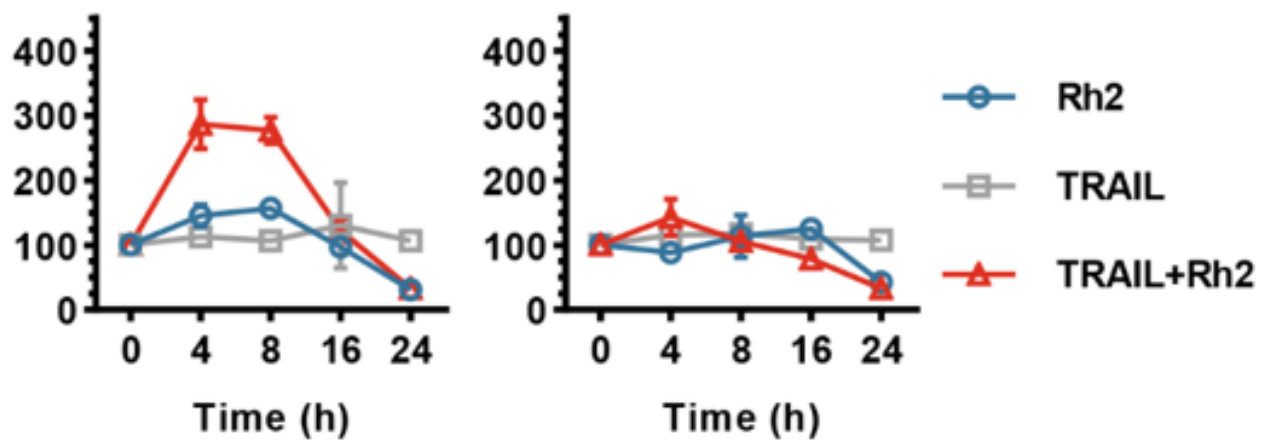

Figure 2

Rh2 enhances the caspase activity induced by TRAIL. 
a
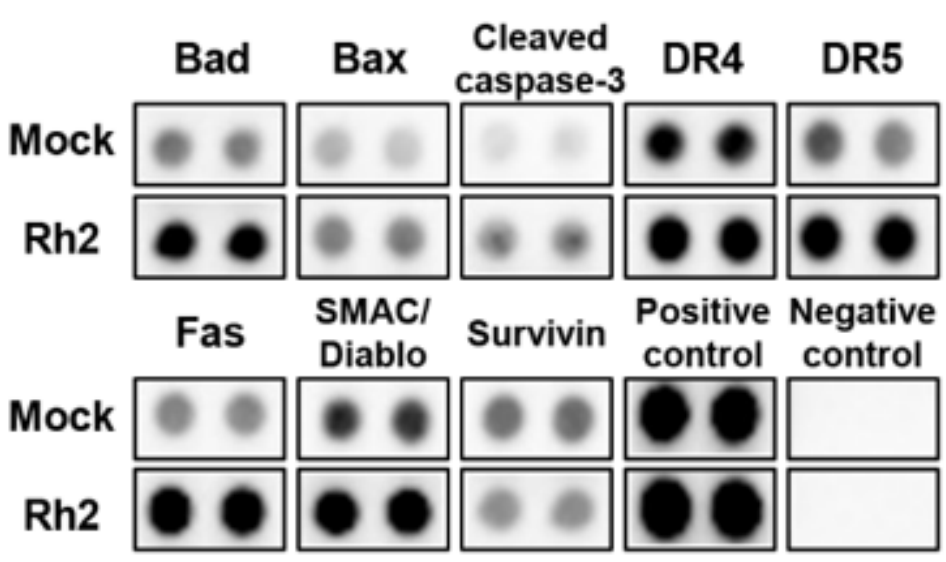

C

\section{Rh2 $\begin{array}{llllllll}0 & 2 & 4 & 8 & 16 & 24 & \mathrm{~h}\end{array}$}
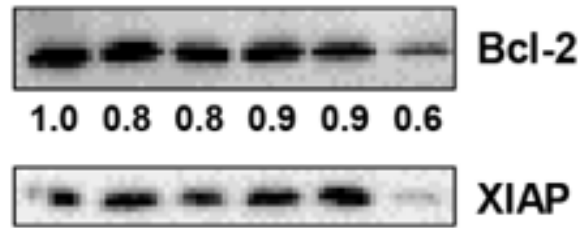

$\begin{array}{llllll}1.0 & 1.3 & 1.1 & 1.3 & 1.4 & 0.7\end{array}$

$\beta$-actin

b

d

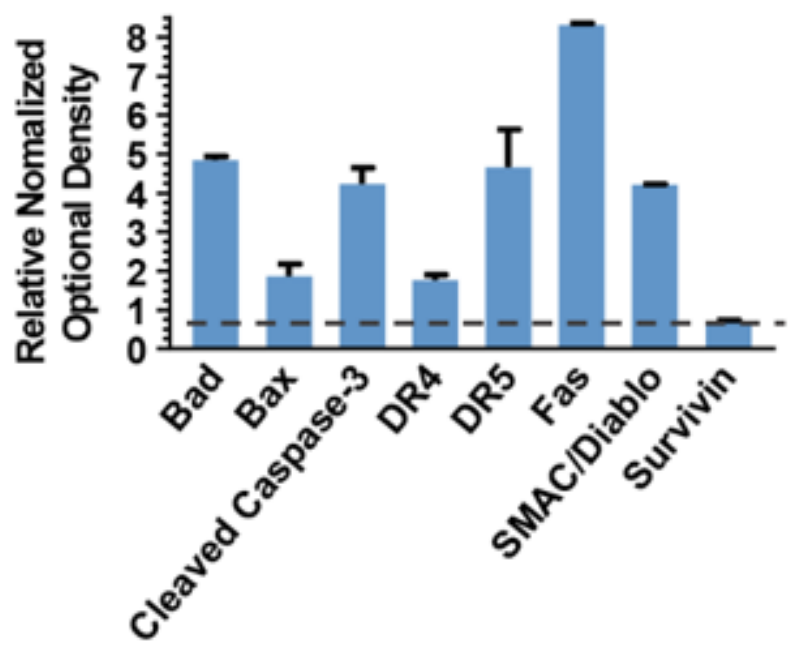

Figure 3

Rh2 downregulates the inhibitor of apoptosis proteins expression. 
a

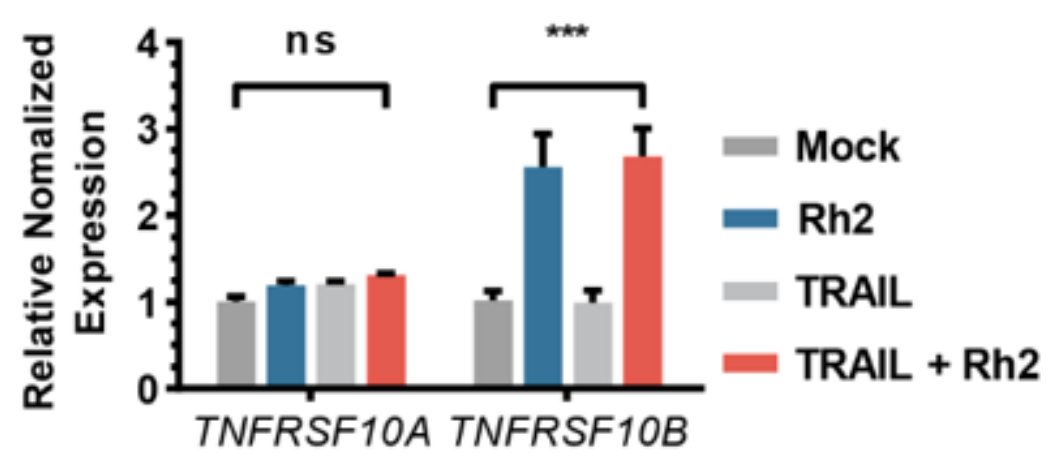

C

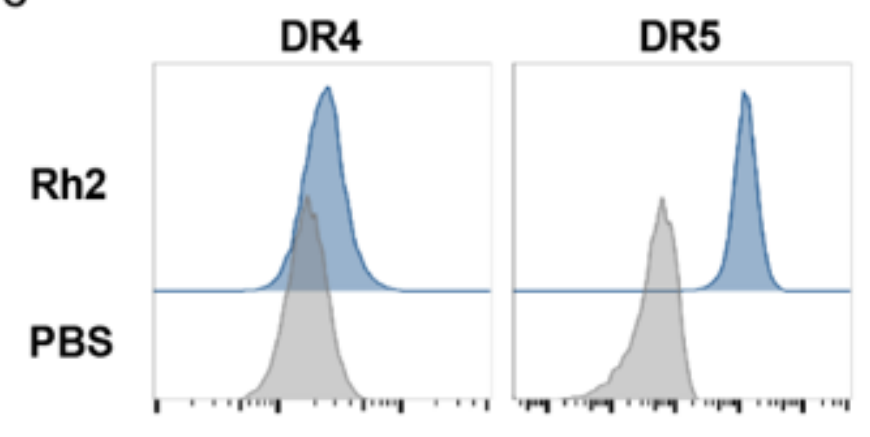

b

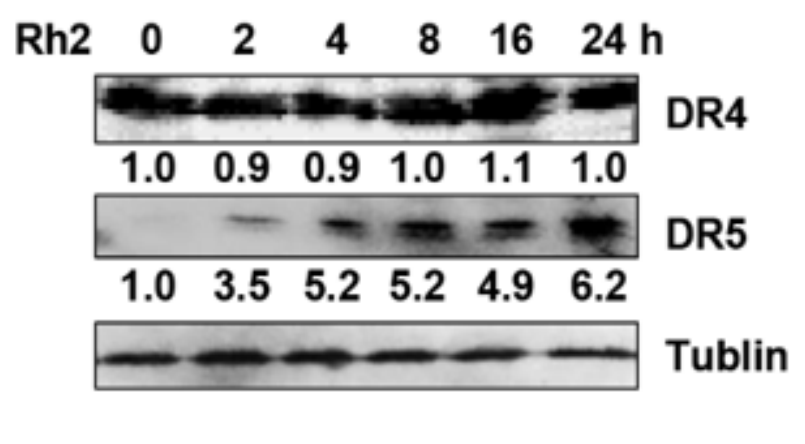

d

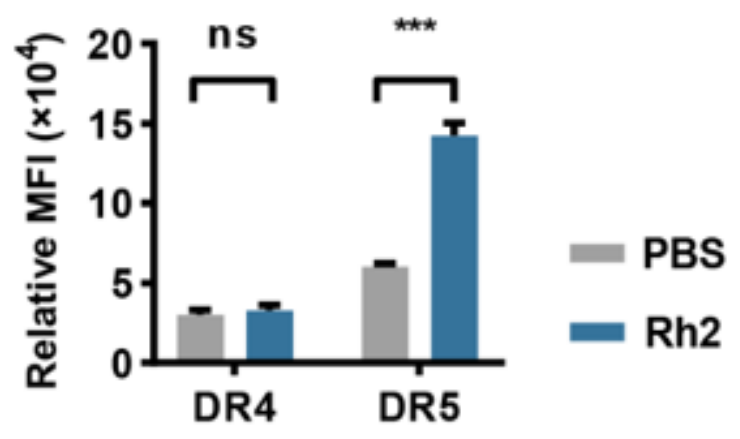

Figure 4

Rh2 upregulates the expression of DR5. 


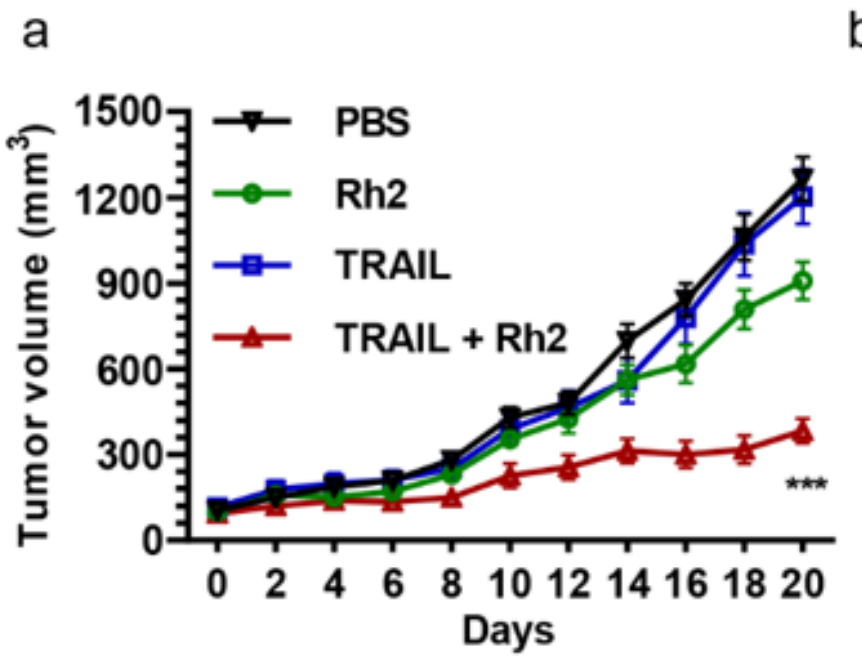

b

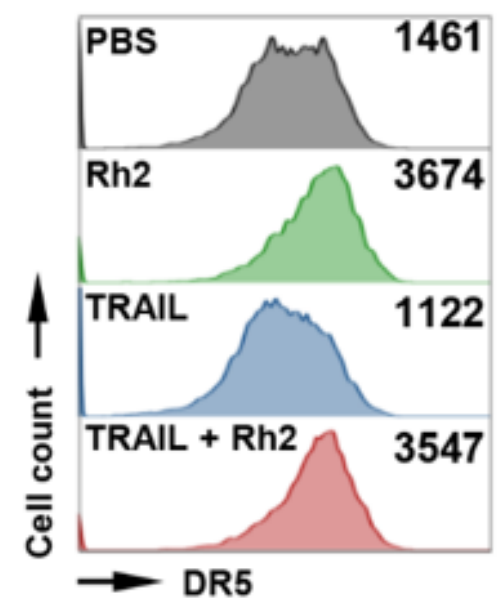

C

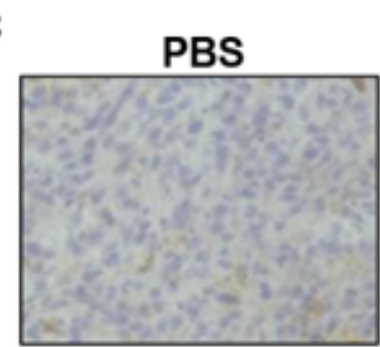

TRAIL
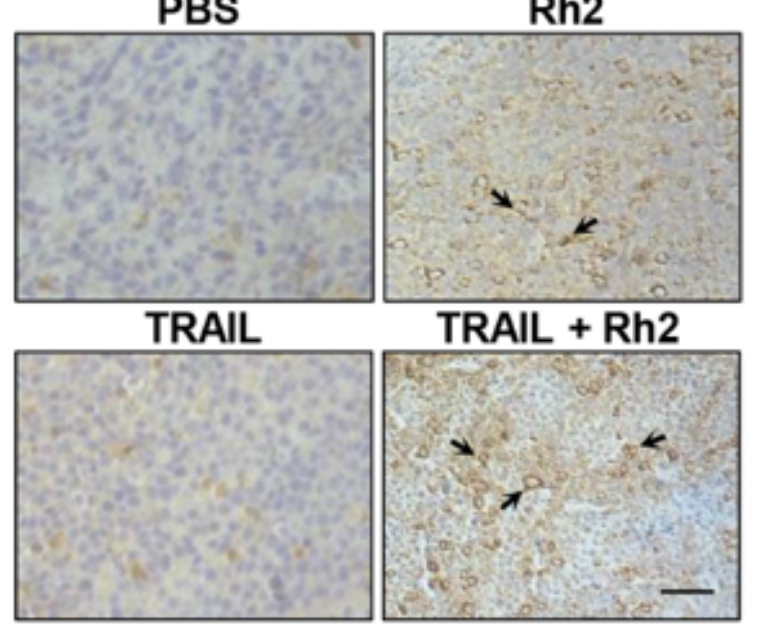

e
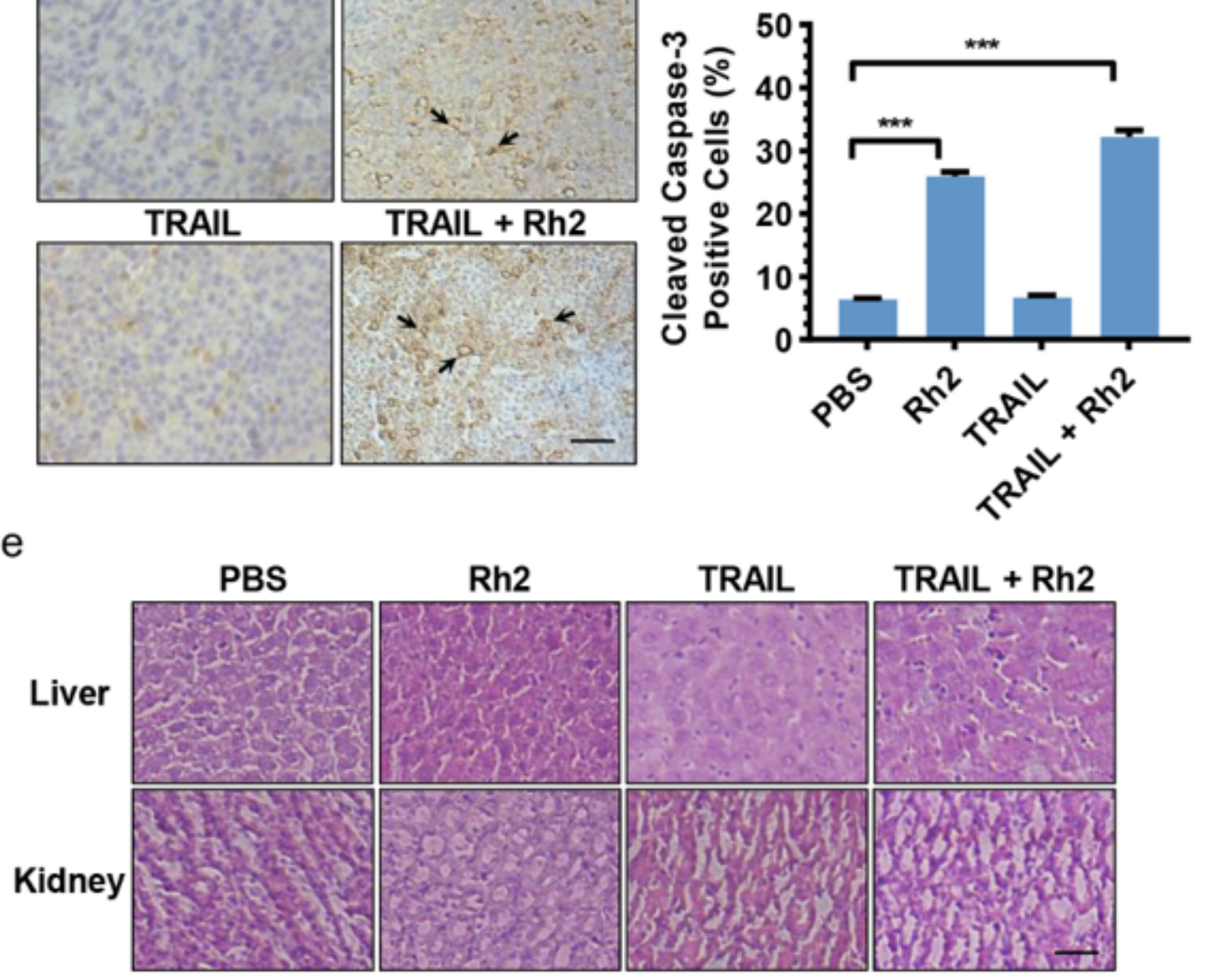

Figure 5

The combination of Rh2 with TRAIL inhibits HepG2 growth in mouse xenografts.

\section{Supplementary Files}

This is a list of supplementary files associated with this preprint. Click to download. 
- Schematic.png

Page $17 / 17$ 